\title{
SeqGene: a comprehensive software solution for mining exome- and transcriptome- sequencing data
}

Xutao Deng

\begin{abstract}
Background: The popularity of massively parallel exome and transcriptome sequencing projects demands new data mining tools with a comprehensive set of features to support a wide range of analysis tasks.

Results: SeqGene, a new data mining tool, supports mutation detection and annotation, dbSNP and 1000 Genome data integration, RNA-Seq expression quantification, mutation and coverage visualization, allele specific expression (ASE), differentially expressed genes (DEGs) identification, copy number variation (CNV) analysis, and gene expression quantitative trait loci (eQTLs) detection. We also developed novel methods for testing the association between SNP and expression and identifying genotype-controlled DEGs. We showed that the results generated from SeqGene compares favourably to other existing methods in our case studies.

Conclusion: SeqGene is designed as a general-purpose software package. It supports both paired-end reads and single reads generated on most sequencing platforms; it runs on all major types of computers; it supports arbitrary genome assemblies for arbitrary organisms; and it scales well to support both large and small scale sequencing projects. The software homepage is http://seqgene.sourceforge.net.
\end{abstract}

\section{Background}

Massively parallel sequencing of exome and transcriptome has been widely adopted to effectively interrogate the key protein-coding and non-coding RNA regions. Exome sequencing (exome-Seq) technology has been especially effective for identifying single-nucleotide polymorphisms (SNPs) and small insertions/deletions (indels) that may cause diseases and other phenotypes. To name a few examples, Ng et al. [1] have found that the mutations of DHODH gene causes Miller syndrome, a Mendelian disorder, by sequencing four affected exomes in three independent kindreds. Yi et al., [2] sequenced 50 exomes of ethnic Tibetans and successfully identified a mutation at EPAS1 gene that is associated with adaptation to high altitude. For quantitative RNA abundance measurement, RNA sequencing (RNA-Seq) compares favourably to other methods, such as gene expression microarrays. The benefits of using RNA-Seq include high resolution,

Correspondence: xutaodeng@gmail.com

Bioinformatics Core Facility, Department of Molecular Medicine, Beckman Research Institute, City of Hope Medical Center, Duarte, CA 91010, USA high dynamic range of expression, low background noise, and the ability to identify allele specific expression and different isoforms [3-6].

However, exome-Seq and RNA-Seq face several bioinformatic challenges, including the development of efficient methods to perform basecalling, assembly, alignment and post-alignment on large amounts of data. There listed more than 350 software tools on http:// seqanswers.com[7] including more than 100 for alignment, more than 50 for sequence assembly, more than 10 for basecalling, and many others for performing various post-alignment analysis tasks. However, most of the post-alignment open source software tools have very limited features and support only one or few analysis tasks. To name a few that relates to our work, ERANGE [8] is a tool for RNA-Seq expression normalization and quantification; SAMtools [9] is mainly developed for alignment format conversion and SNP/indel calling; GAMES [10] supports exome-Seq mutation discovery and functional annotation; DEGseq [11] supports finding differentially expressed genes from RNA-Seq data. Using a combination of software tools for various analytical 
purposes presents a challenge to investigators because the tools often require different hardware specification, operating systems and incompatible data formats. Therefore, there is an urgent need for new exome-Seq and RNA-Seq software tools with a relatively rich feature set that is accessible to investigators with limited or no programming skills to facilitate their multi-analysis requests. We therefore developed SeqGene, an opensource software tool which integrates mutation identification, annotation, genotyping, expression quantification, copy number variation (CNV), expression quantitative trait loci (eQTLs) detection, allele specific expression (ASE), differentially expressed genes (DEGs) identification, and pathway analysis workflows in a single package. SeqGene also implements several novel functions that we proposed, such as a new method for SNP identification and filtering, a new SNP-expression association test based on KEGG-pathways, and a new method for genotype-controlled differentially expressed genes (GCDEG) identification.

\section{Methods}

The major components of SeqGene are illustrated in Figure 1, where the functions were represented in the rectangles, the relationship between them and the corresponding input and out files were shown by arrows, and the file formats are in the red font. Below we explain each major function in more detail.

\section{Mutation detection and annotation}

Detecting genomic variants (such as SNPs, indels and structural variants) via whole-genome sequencing, RNASeq and exome-Seq is an essential approach to understanding the association of genotypic difference to phenotypic consequences with the eventual goal of personalized

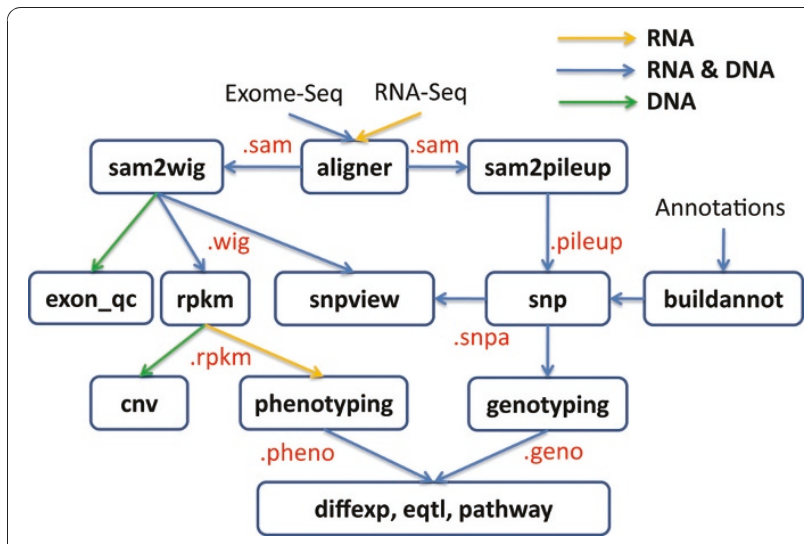

Figure 1 The structure of SeqGene. The diagram shows the relationship between the functions implemented in SeqGene. The rectangles represent the functions; the arrows represent input and output, and the file formats are in the red font. genomics for medical purposes [1,12-15]. Among many open source mutation identification software, SAMtools [9], SNVmix [16], and SOAPsnp [17] are a few widely used ones. SeqGene's mutation detection is implemented in a similar fashion to the pileup function in SAMtools but with a number of new filtering options. To identify SNPs, SeqGene's 'pileup' function reads the alignment results in 'sam' format and reports chromosomal positions for candidate SNPs and indels. From the 'pileup' output, SeqGene's 'snp' function will filter the SNPs and indels based on a number of criteria: 1) the total coverage, i.e., the number of reads covering a candidate SNP (default $20)$; 2) the base quality, when the quality string is present, any base calling with low Phred quality will be removed from the coverage (default 10);3) minor sequenced strand frequency, i.e., the proportion of reads covering both strands must reach certain threshold (default 0.1); and 4) mutated bases frequency, i.e., the proportion of mutated reads must be significant among all reads covering the position (default 0.25).

The multi-criteria SeqGene mutation filter is designed to be versatile to handle various exome-Seq and RNASeq projects. For example, in detecting somatic mutation in cancer samples, one can use a lower allele percentage threshold to account for altered ploidy of cancer samples. In high-depth targeted sequencing, one can increase the coverage threshold to improve the false discovery rate. In addition, since the SNP filter works with 'sam' file, it can work with sequencing data from many sequencing platforms and with various alignment software including Bowtie[18], BWA [19], and Novoalign [20].

\section{Mutation annotation and genotyping}

The 'snp' function also performs mutation annotation such as gene model annotation (upstream, downstream, UTRs, exon, intron, splice sites, etc), miRNA and other non-coding RNA annotation, consequence of the mutation (synonymous, non-synonymous, frame shift, nonsense etc), dbSNP annotation [21], hetero- or homozygosity and ASE on the mutation site. The ASE field lists the number of reads for each allele at all mutation positions. Using RNA-Seq data, users may use the ASE information to detect biased expressed variant alleles on heterozygous coding regions. For human samples, allele frequencies from 1000 Genome [15] data can be added into the annotation as well.

The 'genotyping' function generates genotyping calls on the mutation positions across one or more samples. All positions that pass the SNP filter will be called either 'heterozygous mutations' or 'homozygous mutations'; positions that fail to pass the SNP filter will be labelled 'quality control' for unknown genotypes; positions that are not mutated is called either 'homozygous reference' or 'quality control' depending on whether the coverage 
is above or below the SNP filter threshold. One can use the 'genotyping' function to aggregate mutations across multiple samples to identify mutations that match specific contrasts.

\section{Coverage (sequencing depth) quantification and visualization}

The 'sam 2 wig' function efficiently converts the alignment file into the per-base-resolution coverage file in ". wig' format. For exome-Seq, the 'exon_qc' function report all the missing and defective regions with poor coverage, the quantile of the average exon coverage across exome (coverage sensitivity) and the percentage of total mapped reads aligned onto target exon regions (coverage specificity). For RNA-Seq, the 'rpkm' function output the number of reads covering the genes and estimate the expression abundance using the average coverage as well as RPKM (reads per kilobase of exon model per million mapped reads) [8] as the normalized expression estimation for each transcript and exon. The "phenotyping' function aggregates one or more samples and generates the expression table for all transcripts and exons across all samples. The visualization of coverage and SNPs for each gene can be generated using the 'snpview' function in scalable vector graphics (SVG) format which supports user interactions such as zooming and linking to Ensembl Genome Browser [22].

\section{eQTL}

A quantitative trait locus (QTL) is a region of DNA that is associated with a particular phenotypic trait. eQTLs are genomic loci that regulate expression levels of mRNAs or proteins. By assaying gene expression and genetic variation simultaneously on a genome-wide basis in a large number of individuals, eQTL analysis can map the genetic factors that underpin individual differences in genome-wide gene expression pattern. Detecting eQTLs through RNA-Seq has been demonstrated as a robust and statistically powerful method in recent studies [23-25]. One of the most important applications of eQTL is to combine eQTL detection and genome-wide association (GWA) to identify specific genetic markers that are simultaneously associated with disease and eQTLs, as demonstrated in recent studies in asthma $[26,27]$ and reviewed by Cookson et al. [28]. The 'eqtl' function in SeqGene was computed on expression and genotype data using the ' $I m$ ' function in the $\mathrm{R}$ 'stats' package. The genotype data can be provided by the users or generated from the RNA-Seq data using the 'genotyping' function. In the latter case, the genotyping are limited to those moderately or highly expressed genes on which a sufficient number of reads were mapped for reliable genotyping calls.

\section{Differentially expressed genes (DEGs)}

A common application of RNA-Seq is to identify DEGs between two or more treatment groups. The 'diffexp' function in SeqGene can compute fold change, Student's t-test p-value, Wilcoxon test p-value and false discovery rate (FDR) for all transcripts and exons between two treatment groups. For more complex study designs, one can directly work with the expression table generated using the 'phenotyping' function and the methods borrowed from the microarray gene expression analysis, such as from Bioconductor's limma [29] package, to perform multiple group comparison on RNA-Seq data.

\section{Genotype-controlled differentially expressed genes (GCDEGs)}

A more general way to describe a study design for identifying DEGs is a linear regression model, which describes the linear relationship between treatment variable $t r$ and gene expression variable $e$. For each gene, the linear model is denoted as:

$$
e_{i}=\beta_{0}+\beta \operatorname{tr}_{i}+\varepsilon_{i}, i=1,2, \ldots, n,
$$

where $n$ is the number of samples, $e_{i}$ is the gene expression value of sample $i, t r_{i}$ is the treatment group of sample $i$ (for example, it could be 'treated' or 'control'), $\beta_{0}$ is the intercept parameter, $\beta$ is slope parameter, and $\varepsilon_{i}$ is the error term for sample $i$. This linear model can describe multiple group comparison as well. The test for $\beta \neq 0$ is equivalent to a two-group Student's t-test (if assuming equal variance between the two groups for the Student's t test).

As shown in eQTL studies [23-25], the genotype differences among individuals could significantly impact the overall expression variation. The strong association between genotype and expression, however, could confound and obscure the treatment effect which is the main interests in DEGs. To address this problem, we proposed a new method incorporating genotypes as confounding variables to control for their effects in identifying DEGs in different treatment groups. Suppose a gene harbours $m$ SNPs with its region, the so-called GCDEG is illustrated in a linear regression model as below:

$$
e_{i}=\beta_{0}+\sum_{j=1}^{m} \beta_{j} S N P_{i j}+\beta^{\prime} t r_{i}+\varepsilon_{i}, i=1,2, \ldots, n,
$$

Where $m$ is the number of SNPs within the gene region, $S N P_{i j}$ is the genotype of the $j$ th SNP for the $i$ th sample, $\beta_{j}$ is slope parameter for the $j$ th SNP, $\beta$ ' is slope parameter for treatment after controlling for genotypes. Here we consider only the SNPs in gene regions. The GCDEG strategy is to test both parameter $\beta \neq 0$ in equation (1) and adjusted parameter $\beta^{\prime} \neq 0$ in equation (2) and require both tests to be significant. The genotype information can be 
obtained by other sources. In fact, RNA-Seq data can be used for genotyping moderately to highly expressed genes. In SeqGene, the GCDEG method is implemented in the 'diffexp' function which employs the linear mixed-effects model 'lme' in R package ' $n$ lme'.

\section{Copy number variation}

We implemented an interface in SeqGene to the 'DNAcopy' package in Bioconductor [30] for CNV detection from exome-Seq data. In the ' $c n v$ ' function, the $\log 2$ RPKM estimation of each exon was used as normalized probe signals for chromosomal segmentation and copy number calls. Note that intergenic and intronic CNV calls might not be accurate since these regions are not generally covered by the exome-Seq data. Also note that a reference (such as a normal DNA sample or the average of a group of pooled samples) is needed for absolute copy number calls.

\section{Pathway-based SNP-DEG association}

Detecting significant SNP-expression association using eQTL is effective, however, it requires a large sample size (dozens and above) to generate sufficient statistical power for the genome-wide test. We therefore devised a new pathway topology-based strategy that is especially suited for DEG studies with limited sample size. The assumption of this method is that a SNP-harbouring gene (gSNP) may alter the regulation of the expression of itself and/or a downstream gene (gDEG). The significance of the SNP-DEG association is determined by the topological distance between a gSNP and a gDEG in a regulatory pathway. Therefore a cis-acting SNP (i.e., gSNP and gDEG is the same gene) is considered most significant. The further down the pathway, the less significant of the association. To calculate the distance between any gSNP and gDEG pair, we merge all KEGG pathways [31] graphs into a single directed graph $G$ which contains $N$ genes (nodes). Using Johnson's algorithm [32], we compute the distance matrix $d$ for each pair of genes, where $d_{i, j}$ is the shortest distance from gene $i$ to gene $j$. If there is no path from gene $i$ to gene $j, d_{i, j}$ is set to equal to $N$. The shortest distance from gSNP to gDEG is notated as $d_{g S N P, g D E G}$, which is used as the test statistic for the SNP-DEG association using distance matrix $d$ as the background. The p-value for $d_{g S N P, g D E G}$ is defined by:

$$
\begin{aligned}
& p\left(d_{g S N P, g D E G}\right)=\frac{\sum_{i, j=1}^{N} I\left(d_{i, j} \leqslant d\right)}{N^{2}} \\
& \text { where } \quad I(x) \quad \text { is the indicator function } \\
& I(x)= \begin{cases}1 & \text { if } x=\text { True } \\
0 & \text { if } x=\text { False }\end{cases}
\end{aligned}
$$

\section{Implementation}

SeqGene's major functions (Figure 1) were implemented in Python. Some functions such as CNV, DEG, GCDEG, eQTL and KEGG pathway also require $\mathrm{R}$ and some Bioconductor packages to process their statistical components and graph theory algorithms. The source code is modularly and loosely structured of those components, and therefore, it is relative easy to add new functions to the package. SeqGene supports a simple command-line interface and can also be run in a customized batch processing mode. SeqGene is independent of any specific alignment software; one may choose to use any alignment software as long as the alignment output is in the cross-platform SAM (Sequence Alignment/Map) format [9]. This alignment-independent design allowed SeqGene to support both paired-end reads and single reads generated from most high-throughput sequencing platforms.

SeqGene's algorithms were optimized and one can expect the analysis tasks finish within minutes to a few hours. For example, SeqGene's SNP pileup function runs at similar speed as SAMtools [9] which was implemented in C. The memory fingerprint of SeqGene is well-controlled such that a workstation with 16 G RAM is sufficient for most projects. On multi-processor workstations, one can run multiple jobs of SeqGene to achieve parallel speedup. The annotation packages for latest Ensembl Human, Mouse and Rat [22], and UCSC Genome Browser hg18 and hg19 [33], were pre-built and can be downloaded from the project website. In addition, SeqGene has a function 'buildannot' and corresponding instructions for building additional annotation packages for other organisms from Ensembl, UCSC Genome Browser or arbitrary assemblies.

\section{Results}

Trio-family exome sequencing showed robust SNP identification and genotyping using SeqGene

To test SeqGene's mutation detection algorithm, we performed exome-Seq on a trio family (father, mother, and daughter) with no history of inherited diseases. Genomic DNA was extracted from saliva using Oragene DNA Kit (DNAgenotek Inc., Ontario, Canada) and sonicated using bioruptor (Diagenode Inc., Denville, NJ). Sonicated DNA (3 ug) was used to make a library for paired-end sequencing (Illumina Inc., San Diego, CA) and fragments with approximately $200-250 \mathrm{bp}$ insert DNA were select and amplified. After quality control, $750 \mathrm{ng}$ of the library was hybridized to biotinylated cRNA oligonucleotide baits from the SureSelect Human All Exon kit (Agilent Technologies Inc., Santa Clara, CA), purified by streptavidin-bound magnetic beads, and amplified for 12 cycles. After purification, the library 
was paired-end $(80 \times 80 \mathrm{bp})$ sequenced using Illumina Genome Analyzer IIx (Illumina Inc., San Diego, CA). The exome probes cover $38 \mathrm{Mb}$ of human genome corresponding to the exons and flanking intronic regions of 23,739 genes in the National Center for Biotechnology Information Consensus CDS database (September 2009 release) and also cover 700 miRNAs from the Sanger v13 database and 300 noncoding RNAs from Ensembl GRCh37.56.

The sequencing reads were aligned to Human reference genome (Ensembl GRCh37.56) using Novoalign [20] with default alignment parameters. Mutation identification was performed using SeqGene, SAMtools [9], and VarScan [34] respectively. We used a family-wise SNP filter which ignores any mutations that failed genotyping due to quality control on any of the family members. Table 1 showed the parameters that we used in SeqGene, SAMtools, and VarScan for mutation filters.

Mendelian error rates of the identified SNPs were calculated as an indirect indication of genotyping quality. As demonstrated in Figure 2 and Table 2, SeqGene's mutation identification algorithm had significant lower Mendelian error rates while maintaining similar mutation discovery power comparing with SAMtools. We compared the number of SNPs (after family-wise filter) between VarScan, SAMtools and SeqGene using coverage $>10$ and coverage $>20$ for the three samples, and found that the number of SNPs that passed pedigree check by SeqGene are considerably higher than those by SAMtools for all cases, expect one (Father sample, coverage > 10) where SeqGene identified slightly lower number of SNPs. More importantly, the number of SNPs that failed pedigree check (Mendelian errors) was reduced by around $50 \%$ in SeqGene as compared to SAMtools. For example in Figure 2E, SAMtools identified 72 mutations in the daughter which were not found in any of her parents, whereas SeqGene identified only 12 such Mendelian errors (Figure 2F). SeqGene also compares favorably to VarScan as shown in Table 2 and Figure 2. With similar numbers of identified SNPs, the Mendelian error rates are consistently lower in SeqGene than in VarScan. In addition, the performance of VarScan is consistently better than that of SAMtools.

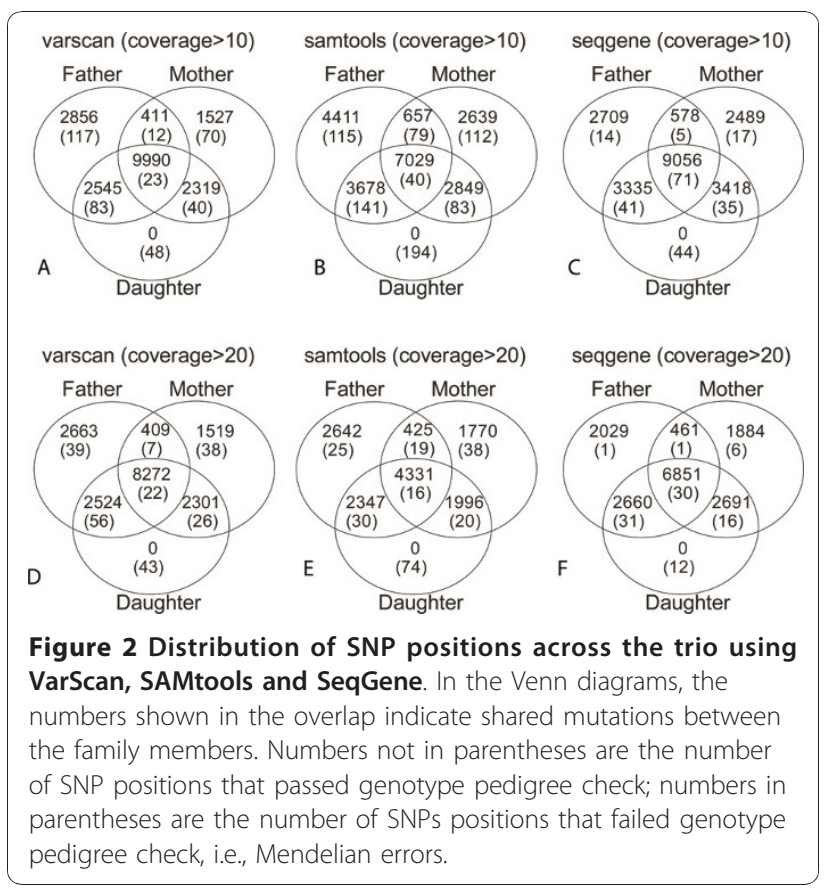

Using the same settings in Table 1, we generated the list of indels using VarScan, SAMtools, and SeqGene respectively and we compared their performance. SeqGene and VarScan consistently outperform SAMtools in terms of Medelian error rates and the number of indels detected as shown in Table 3 and Figure 3. Mixed results were observed when comparing SeqGene and VarScan for indel filtering. Under coverage $>10$, SeqGene generates slightly higher error rate than VarScan. Under coverage $>20$, SeqGene generates lower number of indels than VarScan. However the error rates $(0.4 \%)$ of SeqGene are also lower than those from VarScan (0.9\%-1.2\%).

In addition, SeqGene's 'snp' function can provide detailed annotations to the SNPs based on the gene model categorization (such as 5' UTR, missense, nonsense, intron, splice site, 3' UTR, intergenic, frameshift, synonymous). The resultant annotation file can be aggregated into cross-sample format using 'genotyping' function. Other filtering and analysis with the

Table 1 SNP and indel identification parameters for VarScan, SAMtools and SeqGene in the trio family analysis

\begin{tabular}{|c|c|c|c|}
\hline & VarScan & SAMtools & SeqGene \\
\hline SNP pileup & $\begin{array}{l}\text { SAMtools pileup (default, mapping quality }> \\
\text { 10) }\end{array}$ & $\begin{array}{l}\text { SAMtools pileup (default, mapping quality }> \\
\text { 10) }\end{array}$ & SeqGene pileup (default) \\
\hline SNP filter & $\begin{array}{l}\text { Coverage: }>20,10 \\
\text { Average quality: }>20 \\
\text { Mutated bases frequency: }>25 \% \\
\text { p-value: }<1 \mathrm{E}-6\end{array}$ & $\begin{array}{l}\text { Default filter (SAMtools varfilter) } \\
\text { Coverage: }>20,10 \\
\text { SNP quality }>20\end{array}$ & $\begin{array}{l}\text { Coverage: }>20,10 \\
\text { Bases Phred quality: > } 10 \\
\text { Mutated bases frequency: }>25 \% \\
\text { Minor sequenced strand: }>10 \%\end{array}$ \\
\hline $\begin{array}{l}\text { Family- } \\
\text { wise filter }\end{array}$ & $\begin{array}{l}\text { Ignore positions with at least one 'quality } \\
\text { control' across the family }\end{array}$ & $\begin{array}{l}\text { Ignore positions with at least one 'quality } \\
\text { control' across the family }\end{array}$ & $\begin{array}{l}\text { Ignore positions with at least one 'quality } \\
\text { control' across the family }\end{array}$ \\
\hline
\end{tabular}


Table 2 Number of SNPs and Mendelian error rates using Varscan, SAMtools and SeqGene

\begin{tabular}{ccccccccccc}
\hline & & \multicolumn{3}{c}{ VarScan } & \multicolumn{3}{c}{ SAMtools } & \multicolumn{2}{c}{ SeqGene } \\
\hline & & Father & Mother & Daughter & Father & Mother & Daughter & Father & Mother & Daughter \\
\hline \multirow{2}{*}{$\begin{array}{c}\text { Coverage } \\
>\mathbf{1 0}\end{array}$} & After SNP Filter & 26458 & 19814 & 20788 & 39657 & 24384 & 26971 & 23522 & 22776 & 24097 \\
\hline & After Family Filter & 16037 & 14392 & 15048 & 15775 & 13174 & 13556 & 15678 & 15541 & 15809 \\
& & $(235)$ & $(145)$ & $(194)$ & $(375)$ & $(324)$ & $(458)$ & $(131)$ & $(128)$ & $(191)$ \\
\hline & Mendelian Error Rate (\%) & $\mathbf{1 . 5}$ & $\mathbf{1 . 0}$ & $\mathbf{1 . 3}$ & $\mathbf{2 . 3}$ & $\mathbf{2 . 4}$ & $\mathbf{3 . 6}$ & $\mathbf{0 . 8}$ & $\mathbf{0 . 8}$ & $\mathbf{1 . 2}$ \\
\hline $\begin{array}{c}\text { Coverage } \\
>\mathbf{2 0}\end{array}$ & After SNP Filter & 23444 & 16317 & 17639 & 27814 & 15797 & 18060 & 18805 & 16354 & 17889 \\
\hline & After Family Filter & 13992 & 12594 & 13244 & 9745 & 8522 & 8674 & 12001 & 11887 & 12202 \\
& & $(124)$ & $(93)$ & $(147)$ & $(90)$ & $(93)$ & $(140)$ & $(63)$ & $(53)$ & $(89)$ \\
\hline
\end{tabular}

annotation files are possible. For example, the users can obtain non-synonymous mutations using 'polyphen' function and the output can be submitted to PolyPhen server [35] for further processing.

\section{Identify eQTLs in HapMap RNA-Seq data}

In this example, we showed the SeqGene's capability on expression quantification and eQTL by reanalyzing a public data set from the international HapMap project $[23,36]$. The data set contains the RNA-Seq samples of $60 \mathrm{CEU}$ individuals (HapMap individuals of European descent). The mRNA fraction of the transcriptome of lymphoblastoid cell lines (LCLs) from those samples were sequenced using 37-base pairs (bp) paired-end Illumina sequencing. Each individual's transcriptome was sequenced in one lane of an Illumina GAII analyzer.

We aligned the short reads to the UCSC Genome Browser hg19 human reference genome [33] using Tophat [37], which can automatically detect and align the short reads to candidate exon-exon junctions. We use SeqGene's 'sam2wig' and 'rpkm' functions to quantify gene expression of individual samples. SeqGene's 'phenotyping' function is then used to tabulate gene expression across multiple RNA-Seq samples. The genotype information was obtained from the international HapMap project [36]. The expression profiles from multiple samples, along with the genotypes, were processed using SeqGene's 'eqtl' function, which is capable to report both cis- (locally) and trans- (at a distance) eQTLs to a gene. Figure 4 showed an example of a strong eQTL that affects the expression level of gene KB-1839H6.1. The genetic marker is dbSNP entry rs1042927, which is located on chromosome 11, whereas the gene KB-1839H6.1 is located on chromosome 22. Therefore, this is a trans-eQTL which maps far from the location of its gene-of-origin gene. The Bonferroniadjusted p-value of this eQTL is 1.39e-5. The 'snpview' function in SeqGene will further display the wiggle plot superimposed on the gene model, as shown in Figure 4.

\section{Identify GCDEGs from public RNA-Seq dataset}

We demonstrate the novel GCDEG method in SeqGene by reanalyzing a recently published RNA-Seq dataset [38]. The samples contain double poly(A)-selected RNA from primary $\mathrm{CD} 4+\mathrm{T}$ cells with both activated and untreated conditions. We aligned the short reads to the UCSC Genome Browser hg19 human reference sequences [33] using Tophat [37]. The genome-wide gene expression profiling were performed using 'sam2wig', 'rpkm', and 'phenotyping' functions. Then the 'diffexp' function was used to perform two-group comparison between the 'stimulated' and 'unstimulated' samples to identify DEGs and GCDEGs. DEGs were selected using Student's t-test p-value $<0.01$. GCDEGs were selected using two cutoff values: Student's t-test pvalue $<0.01$ and genotype-controlled $\mathrm{p}$-value $<0.01$.

Table 3 Number of indels and Mendelian error rates using Varscan, SAMtools and SeqGene

\begin{tabular}{|c|c|c|c|c|c|c|c|c|c|c|}
\hline & & \multicolumn{3}{|c|}{ VarScan } & \multicolumn{3}{|c|}{ SAMtools } & \multicolumn{3}{|c|}{ SeqGene } \\
\hline & & Father & Mother & Daughter & Father & Mother & Daughter & Father & Mother & Daughter \\
\hline & After SNP Filter & 696 & 522 & 522 & 539 & 560 & 634 & 637 & 628 & 703 \\
\hline \multirow[t]{3}{*}{$\begin{array}{c}\text { Coverage } \\
>10\end{array}$} & After Family Filter & $\begin{array}{c}356 \\
(4)\end{array}$ & $\begin{array}{c}330 \\
(4)\end{array}$ & $\begin{array}{c}331 \\
(3)\end{array}$ & $\begin{array}{c}279 \\
(5)\end{array}$ & $\begin{array}{c}279 \\
(4)\end{array}$ & $\begin{array}{c}278 \\
(8)\end{array}$ & $\begin{array}{c}320 \\
(4)\end{array}$ & $\begin{array}{l}318 \\
(3)\end{array}$ & $\begin{array}{c}322 \\
(6)\end{array}$ \\
\hline & Mendelian Error Rate (\%) & 1.1 & 1.2 & 0.9 & 1.8 & 1.4 & 2.9 & 1.2 & 0.9 & 1.9 \\
\hline & After SNP Filter & 690 & 503 & 506 & 389 & 355 & 413 & 478 & 416 & 472 \\
\hline \multirow[t]{2}{*}{$\begin{array}{c}\text { Coverage } \\
>20\end{array}$} & After Family Filter & $\begin{array}{l}340 \\
(4)\end{array}$ & $\begin{array}{l}316 \\
(4)\end{array}$ & $\begin{array}{c}319 \\
(3)\end{array}$ & $\begin{array}{l}180 \\
(2)\end{array}$ & $\begin{array}{l}182 \\
(2)\end{array}$ & $\begin{array}{l}178 \\
(1)\end{array}$ & $\begin{array}{c}223 \\
(1)\end{array}$ & $\begin{array}{l}223 \\
(1)\end{array}$ & $\begin{array}{c}223 \\
(1)\end{array}$ \\
\hline & Mendelian Error Rate (\%) & 1.2 & 1.3 & 0.9 & 1.1 & 1.1 & 0.6 & 0.4 & 0.4 & 0.4 \\
\hline
\end{tabular}




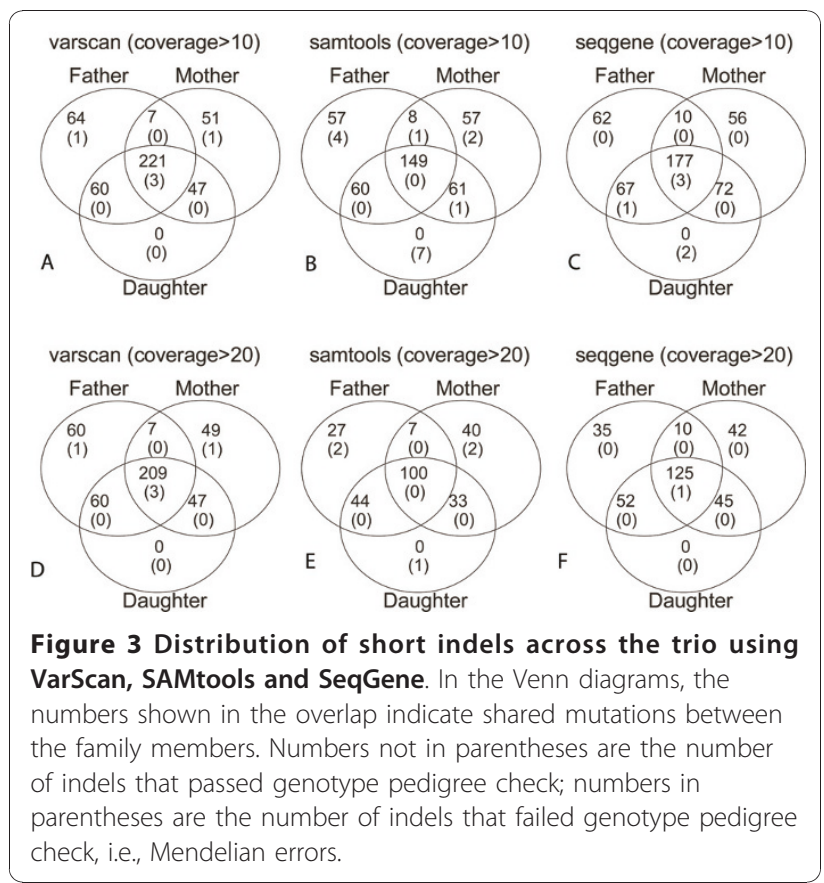

We compared the variance components on the selected DEGs and GCDEGs using SeqGene by the'varcomp' function in $\mathrm{R}$ package 'ape'. Figure 5A showed the variance components for DEGs and GCDEGs for the 'treatment', 'genotype' and 'residue' components, respectively. We observed significant residual error reduction in the GCDEGs method as compared to DEGs, and more variance was explained by the 'genotype' component in the GCDEGs. Figure 5B showed an example gene in which the treatment effect is badly confounded with genotype. This example illustrated that GCDEGs can help reduce errors and avoid DEGs that are confounded with genotype.

\section{Identify somatic mutation and copy number variation from Acute Myeloid Leukemia (AML) exome sequencing data}

We reanalysed the exome sequencing data from a recent study by Yan et al. [39]. The dataset contains nine paired samples of AML-M5 cases with bone marrow cancer samples obtained at the time of diagnosis and control peripheral blood specimens obtained after complete remission. Five additional AML-M5 cases without matched normal samples were also analyzed. The captured target in each exome was $24 \mathrm{Mb}$. From EBI sequence Read Archive with submission ID SRP005624, we downloaded a total of 96 lane of sequencing runs in. fastq format and aligned the reads to Human hg19 reference assembly using bwa [19]. Table 4 shows the alignment coverage report using 'exon_qc' function for the nine bone marrow samples and their corresponding

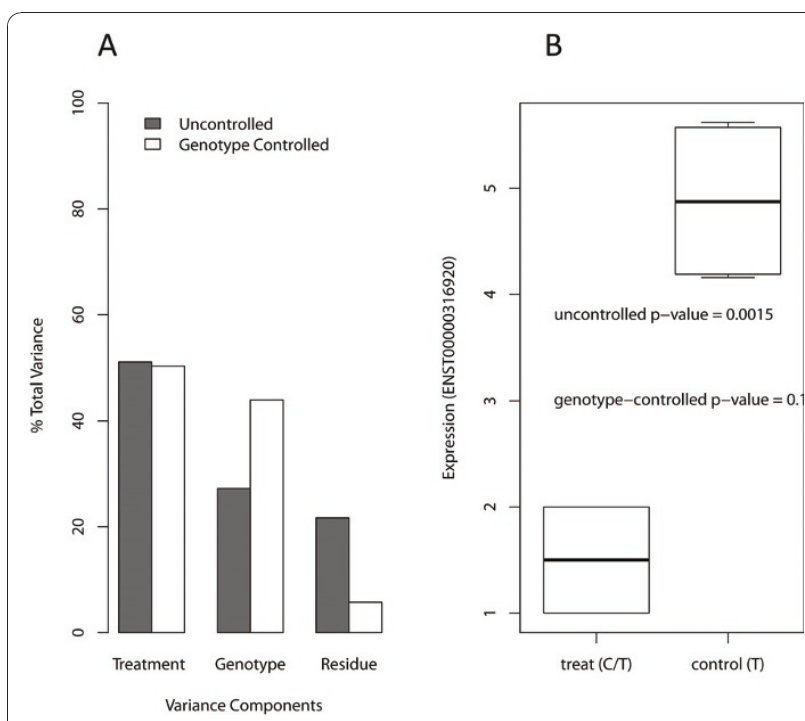

Figure 5 Identifying GCDEGs using SeqGene on RNA-Seq data. (A). Comparing variance components of GCDEGs (white bars) with DEGs (dark bars). The variance components were computed for three factors (Treatment, Genotype, and Residue) using SeqGene with the 'Ime' function in R package ' $n / m e^{\prime}$ and the 'varcomp' function in R package 'ape'. In this example, the uncontrolled DEGs were detected using Student's t-test $p<0.01$. The controlled GCDEGs satisfied both Student's t-test $p<0.01$ and the adjusted $p$ value $<0.01$. We observed that Residue variance is significantly reduced in GCDEGs. (B). An example gene showing the effect of avoiding the confounding genotype factor. In this example, the treatment and genotype are completely confounded in that the treatment samples had genotype (C/T) and the control samples had genotype $(T / T)$. The uncontrolled $p$-value is 0.0015 , whereas it is no longer significant after genotype controlling.

blood samples. The average coverage for the samples is in the range 44 fold to 117 fold on refseq exons. $61 \%$ to $68 \%$ of exons in refseq were covered at $>10$ fold on average. $65 \%$ to $70 \%$ of exons in refseq were covered with $>5$ fold on average. We next carried out mutation detection and filtering using seqgene's 'sam2wig', 'sam2pileup', 'snp' and 'genotyping' functions to obtain the genotype in a tabulated format across 23 samples. We obtained rare somatic mutations in bone marrow sample by filtering dbSNP 131 and the germline mutations in blood sample. Table 5 lists three rare somatic missense mutations for DNA methyltransferase gene DNMT3A which is consistent with the original report. Note that one mutation may be located at multiple transcripts and therefore was annotated multiple times.

We then performed CNV analysis on the nine pairs of samples using the 'cnv' function. For each cancer sample, its control blood sample was used to normalize the signals. The 'cnv' function generated results in '. seg' format which include genomic break points estimation and mean signals for all genomic regions. The output 'seg' file was loaded in to Integrative Genomics 
A

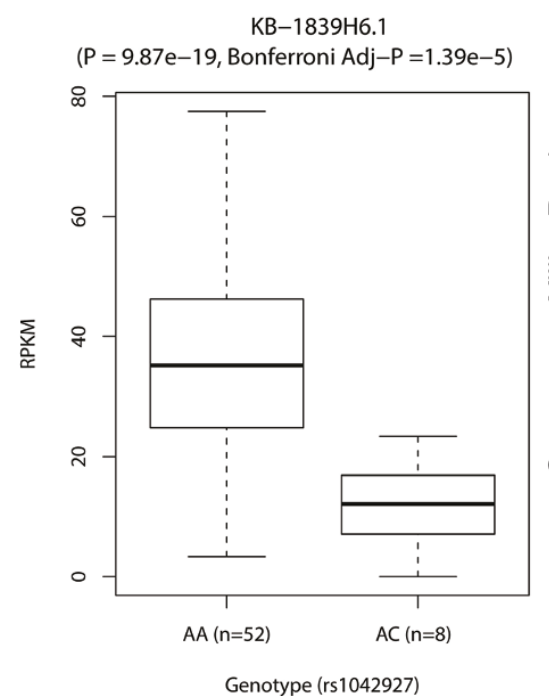

B

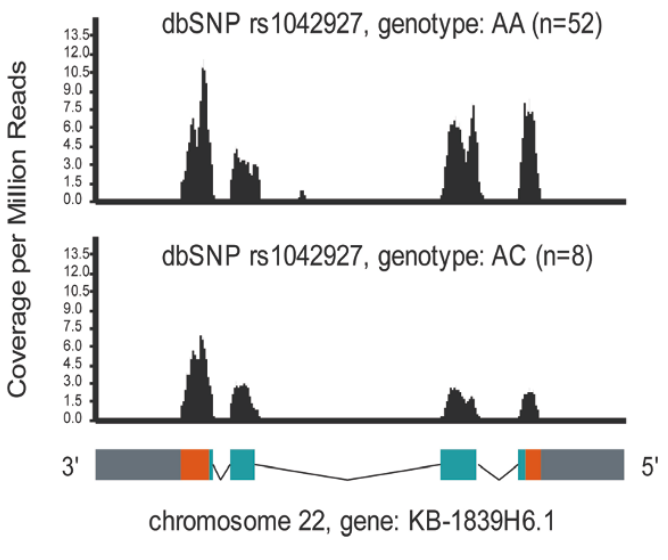

Figure 4 An example of significant eQTL identified using SeqGene. The HapMap individuals were stratified based on their genotypes at rs 1042927 (dbSNP entry nubmer). We found 52 individuals are of genotype 'AA' and the other eight samples are of genotype 'AC'. We compared the expression levels of the gene KB-1839H6.1 in the two groups. (A). The KB-1839H6.1 gene expression level (RPKM) in the two groups (with 'AA' or 'AC' genotype at rs 1042927). The expression levels were quantified using SeqGene's 'rpkm' function. (B). The aggregated coverage in the two groups illustrated on the gene model. The coverage is normalized to 'Coverage per Million Reads'. This plot was generated using the SeqGene's 'snpview' function. Gene models for every transcript were displayed at the bottom as flanking regions (gray), UTRs (orange), CDS (green) and introns (lines).

Viewer (IGV) [40] for visualization and the results were shown in Figure 6. From the copy number aggregation view (Figure 6A), we observed recurrent (more than 2 cases) copy number gain on chromosome $5 \mathrm{q}$, $17 q 25,19$, and 22. Particularly, four out of the nine samples show amplification on significant portions of chromosome 19. This results indicate chromosome 19 amplifications may be a hallmark of AML as reported in an earlier study by Nimer et al. [41]. It should be noted that exome-Seq experiments focus only on exons and generate very uneven coverage across exons due partially to sequence capture biases. However, analysis using exome-Seq data may still shed light on copy number variations beyond the exons when paired control samples are available and a reasonable breakpoint estimation algorithm such as Circular Binary Segmentation (CBS) [30] is used.

We also recorded run time and memory usage when performing different tasks for this relatively large scale project (Table 6). Note that currently all tasks can finish in reasonable time except for global trans-eQTL calculation which needs days of calculation using on multiple CPUs.

\section{Comparing mutations discovered using paired exome-Seq and RNA-Seq samples}

We reanalyzed paired RNA-Seq and exome-Seq data derived from breast cancer cell line, HCC1954 reported

Table 4 Quality control of AML samples annotated on refseq, Human hg19

\begin{tabular}{llllllllll}
\hline & Blood1 & Blood2 & Blood3 & Blood4 & Blood5 & Blood6 & Blood7 & Blood8 & Blood9 \\
\hline mde & 48 & 47 & 50 & 47 & 87 & 86 & 99 & 102 & 95 \\
\hline ec5 (\%) & 65 & 68 & 67 & 69 & 69 & 69 & 69 & 69 & 69 \\
\hline ec10(\%) & 61 & 65 & 65 & 66 & 66 & 66 & 67 & 67 & 67 \\
\hline mde & Bone1 & Bone2 & Bone3 & Bone4 & Bone5 & Bone6 & Bone7 & Bone8 & Bone9 \\
\hline ec5 (\%) & 66 & 45 & 44 & 44 & 79 & 86 & 75 & 77 & 117 \\
\hline ec10(\%) & 65 & 68 & 68 & 69 & 69 & 69 & 68 & 68 & 70 \\
\hline
\end{tabular}


Table 5 Three novel missense somatic mutations of DNMT3A identified in 23 samples using seqgene (cov > 10)

\begin{tabular}{|c|c|c|c|c|c|c|c|c|c|c|}
\hline transcript & position (hg19) & $\begin{array}{l}\text { position } \\
\text { transcript }\end{array}$ & codon number & amino acid change & ref & bl3 & bm3 & bl9 & bm9 & ex5 \\
\hline NM_022552 & chr2:25457197 & 2947 & 897 & Val- > Asp & $A$ & A & $\mathrm{A} / \mathrm{T}$ & & & \\
\hline NM_175629 & chr2:25457197 & 3028 & 897 & Val- > Asp & A & A & $\mathrm{A} / \mathrm{T}$ & & & \\
\hline NM_153759 & chr2:25457197 & 2237 & 708 & Val- > Asp & A & A & $\mathrm{A} / \mathrm{T}$ & & & \\
\hline NM_022552 & chr2:25467449 & 1884 & 543 & Gly- > Cys & C & & & & & $A / C$ \\
\hline NM_175629 & chr2:25467449 & 1965 & 543 & Gly- > Cys & C & & & & & $A / C$ \\
\hline NM_153759 & chr2:25467449 & 1174 & 354 & Gly- > Cys & C & & & & & $\mathrm{A} / \mathrm{C}$ \\
\hline NM_022552 & chr2:25457242 & 2902 & 882 & Arg- $>$ His & C & & & $\mathrm{C} / \mathrm{T}$ & C & \\
\hline NM_175629 & chr2:25457242 & 2983 & 882 & Arg- $>$ His & $C$ & & & $\mathrm{C} / \mathrm{T}$ & C & \\
\hline NM_153759 & chr2:25457242 & 2192 & 693 & Arg- $>$ His & C & & & $\mathrm{C} / \mathrm{T}$ & C & \\
\hline
\end{tabular}

by Zhao et al. [42]. Our goal of this integrated analysis is to estimate the correlation between the mutations identified using paired DNA and RNA sequencing of cancer samples. Exome-Seq was performed on Roche 454 platform and RNA-Seq was performed on Illumina GAII platform. The datasets were downloaded from the EBI Sequence Read Archive (ERA) with submission ID ERA010917 for exome-Seq data and ERA011762 for RNA-Seq data.
We applied Tophat [37] for the RNA-Seq spliced alignment and bwa-sw [19] for exome-Seq long reads alignment on Human hg19 assembly. We generated quality control reports using 'exon_qc' function on the two samples respectively. The exome-Seq alignment shows that $59.6 \%$ of the refseq exons were covered at $\geq$ 5 fold and the mean coverage on all exons is 24 fold; RNA-Seq yields $4.8 \%$ of the refseq exons with $\geq 5$ fold coverage and the mean coverage on all exons is only 1.9

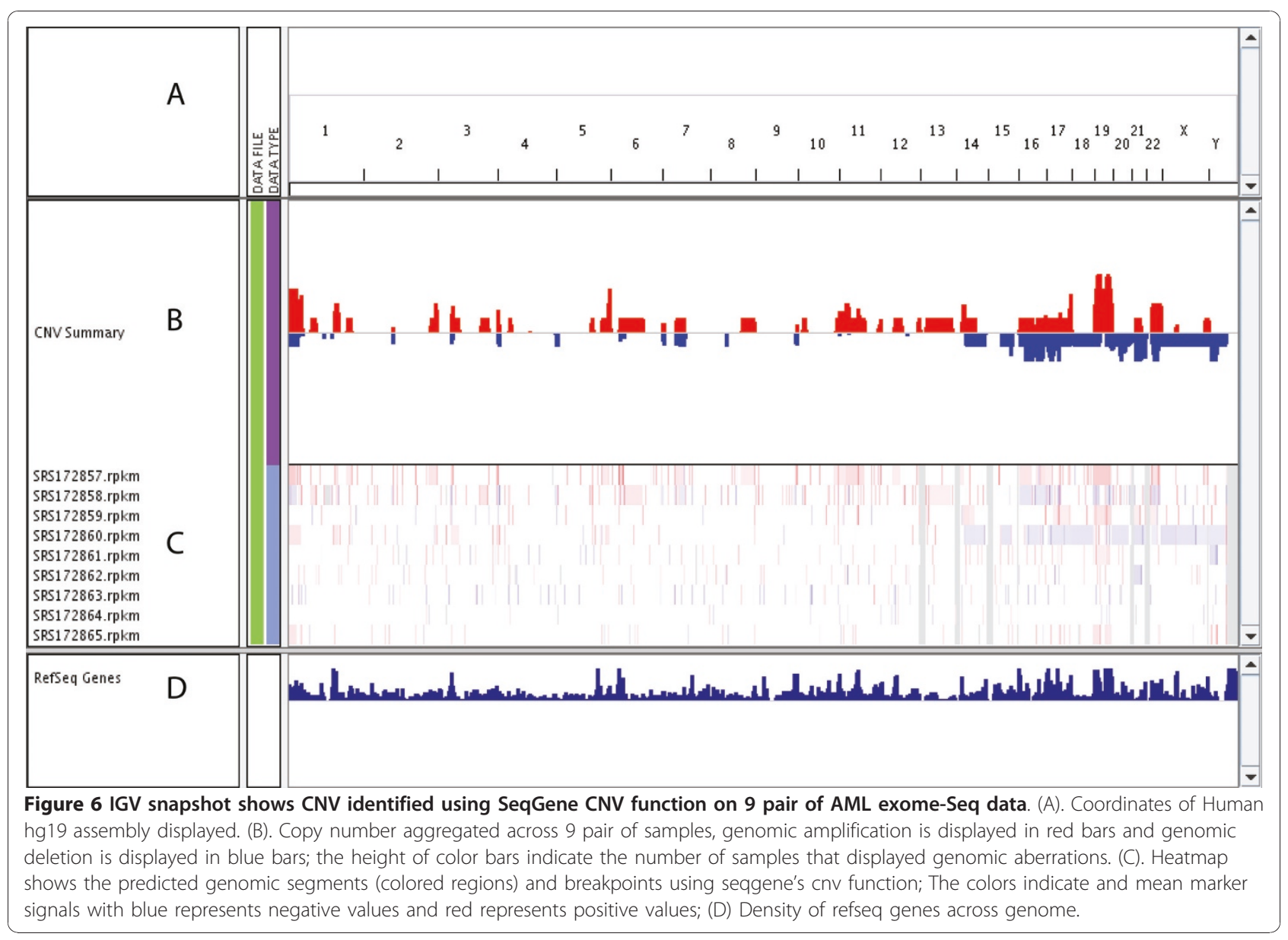


Table 6 Computing performance of major functions using 23 exome sequencing samples on a 16 CPU workstation

\begin{tabular}{llllll}
\hline Script & Function & \#CPU & $\begin{array}{l}\text { Peak RAM } \\
(\mathbf{G b})\end{array}$ & Time & Notes \\
\hline exon_qc & quality control & 1 & 6 & $10-20$ min & Per sample \\
\hline sam2wig & generate wig file & 1 & 2 & $20-50$ min & Per sample \\
\hline sam2pileup & generate pileup file & 1 & $3-7$ & $1-4$ hours & Per sample \\
\hline snp & annotate and filter snp and indels & 1 & 4 & $2-8$ min & Per sample \\
\hline rpkm & quantify coverage on gene model & 1 & 4 & $4-16$ min & Per sample \\
\hline cnv & copy number variation & 1 & 4 & 5 min & Per sample \\
\hline genotyping & genotyping file across samples & 1 & 8 & 4 hour & Across 23 samples \\
\hline phenotyping & coverage (expression) across samples & 1 & 5 & 10 min & Across 23 samples \\
\hline eqtl -m cis & Cis- EQTL & 1 & 3 & 1 hour & 1000 genome data \\
\hline eqtl -m trans & Trans-EQTL & $16 *$ & 3 & 7 days & 1000 genome data \\
\hline
\end{tabular}

fold. Quality control of the RNA sample shows that $3.4 \%$ of the 48 million aligned reads were located on intergenic regions or introns, indicating possible contamination of the RNA sample. We then performed mutation discovery on the paired samples using SeqGene. After applying quality control filtering to obtain SNPs that passed quality control in both exome and RNA samples, we identified 29 the SNPs on coding regions and UTRs. We then compared the genotypes of the 29 SNPs between exome and RNA for genotyping consistency. The results were summarized in Table 7 . The total number of matched mutations between exome and RNA samples is 20 out of the 29 SNPs. Five heterozygous SNPs (called from DNA) showing homozygous expression pattern from RNA are ASE candidates. Three homozygous SNPs (called from DNA) showing heterozygous expression on RNA sample are obvious genotyping inconsistencies. The lower than expected number of SNPs and low genotyping consistency between exome and RNA genotyping may be due to a number of factors such as biased exome sequence capture, possible contamination of RNA sample, misplaced alignment, and sequencing errors.

\section{Conclusion}

We developed an open-source software tool, SeqGene, to support massively parallel exome-Seq and RNA-Seq data analysis. SeqGene supports functions of base-resolution read coverage, quality control, SNP/indel

Table 7 Number of SNPs between paired RNA-Seq and Exome-Seq samples

\begin{tabular}{ccc}
\hline & RNA-hom & RNA-het \\
\hline DNA-hom & $17(0)^{c}$ & $3^{a}$ \\
\hline DNA-het & $5^{b}$ & $3(1)^{c}$ \\
\hline
\end{tabular}

a:genotyping inconsistency between DNA and RNA, b: candidate ASE, c: number of inconsistent genotypes identification and annotation, RNA and DNA depth quantification, ASE, CNV, eQTL, DEG, and KEGG pathway analysis. Among the many functions of SeqGene, we have also implemented novel methods for genotype-controlled differentially expressed genes (GCDEGs) identification, and SNP-DEG association test using KEGG pathways. We have demonstrated that SeqGene is a useful data mining tool to support a wide variety of analysis tasks in exome-Seq and RNA-Seq data.

\section{Availability and requirements}

The SeqGene software, annotation packages and user's manual can be accessed at http://seqgene.sourceforge. net. SeqGene requires Python 2.6 or 2.7 and CNV, DEG, GCDEG, eQTL and KEGG pathway functions also require $R$ and certain Bioconductor packages. SeqGene is cross-platform software and has been tested on Linux-, Macintosh- and Windows- based workstations. SeqGene is free for academic use and require a license from the author for commercial applications.

\begin{abstract}
Abbreviations
AML: acute myeloid leukemia; ASE: allele specific expression; CBS: circular binary segmentation; CNV: copy number variation; DEG: differentially expressed gene; eQTL: expression quantitative trait locus; FDR: false discovery rate; GCDEG: genotype-controlled differentially expressed gene; GWA: genome-wide association; IGV: integrative genomics viewer; LCL: lymphoblastoid cell lines; miRNA: MicroRNA; QTL: quantitative trait locus; RPKM: reads per kilobase of exon model per million mapped reads; SNP: single nucleotide polymorphism; SVG: scalable vector graphics; UTR: untranslated regions.
\end{abstract}

\section{Acknowledgements}

The author would like to thank Dr. Harry Gao, Dr. Yate-Ching Yuan, Dr. Kun Qu and Dr. Richard Jove of City of Hope for providing testing data and computing facilities for the trio family exome-Seq study. The author would also like to thank the reviewers and software users for their comments.

Authors' contributions

XD designed and implemented the SeqGene, performed the analysis and wrote the manuscript. 


\section{Competing interests}

The authors declare that they have no competing interests.

Received: 14 January 2011 Accepted: 29 June 2011

Published: 29 June 2011

\section{References}

1. Ng SB, Buckingham KJ, Lee C, Bigham AW, Tabor HK, Dent KM, Huff CD, Shannon PT, Jabs EW, Nickerson DA, Shendure J, Bamshad MJ: Exome sequencing identifies the cause of a mendelian disorder. Nat Genet 2010, 42:30-35.

2. Yi X, Liang Y, Huerta-Sanchez $E$, Jin $X$, Cuo ZX, Pool JE, Xu X, Jiang $H$, Vinckenbosch N, Korneliussen TS, Zheng H, Liu T, He W, Li K, Luo R, Nie X, Wu H, Zhao M, Cao H, Zou J, Shan Y, Li S, Yang Q, Ni P, Tian G, Xu J, Liu X, Jiang $T$, Wu $R$, et al: Sequencing of 50 human exomes reveals adaptation to high altitude. Science 2010, 329:75-78.

3. Cloonan N, Forrest AR, Kolle G, Gardiner BB, Faulkner GJ, Brown MK, Taylor DF, Steptoe AL, Wani S, Bethel G, Robertson AJ, Perkins AC, Bruce SJ, Lee CC, Ranade SS, Peckham HE, Manning JM, McKernan KJ, Grimmond SM: Stem cell transcriptome profiling via massive-scale mRNA sequencing. Nat Methods 2008, 5:613-619.

4. Lister R, O'Malley RC, Tonti-Filippini J, Gregory BD, Berry CC, Millar AH, Ecker JR: Highly integrated single-base resolution maps of the epigenome in Arabidopsis. Cell 2008, 133:523-536.

5. Marioni JC, Mason CE, Mane SM, Stephens M, Gilad Y: RNA-seq: an assessment of technical reproducibility and comparison with gene expression arrays. Genome Res 2008, 18:1509-1517.

6. Wilhelm BT, Marguerat S, Watt S, Schubert F, Wood V, Goodhead I, Penkett CJ, Rogers J, Bahler J: Dynamic repertoire of a eukaryotic transcriptome surveyed at single-nucleotide resolution. Nature 2008, 453:1239-1243.

7. [http://seganswers.com/wiki/Software].

8. Mortazavi A, Williams BA, McCue K, Schaeffer L, Wold B: Mapping and quantifying mammalian transcriptomes by RNA-Seq. Nat Meth 2008, 5:621-628

9. Li H, Handsaker B, Wysoker A, Fennell T, Ruan J, Homer N, Marth G, Abecasis G, Durbin R, Genome Project Data Processing Subgroup: The Sequence Alignment/Map format and SAMtools. Bioinformatics 2009, 25:2078-2079.

10. Sana ME, lascone M, Marchetti D, Palatini J, Galasso M, Volinia S: GAMES identifies and annotates mutations in next-generation sequencing projects. Bioinformatics

11. Wang L, Feng Z, Wang X, Wang X, Zhang X: DEGseq: an R package for identifying differentially expressed genes from RNA-seq data. Bioinformatics 2010, 26:136-138.

12. Kim Jl, Ju YS, Park H, Kim S, Lee S, Yi JH, Mudge J, Miller NA, Hong D, Bell CJ, Kim HS, Chung IS, Lee WC, Lee JS, Seo SH, Yun JY, Woo HN, Lee H, Suh D, Kim HJ, Yavartanoo M, Kwak M, Zheng Y, Lee MK, Kim JY, Gokcumen O, Mills RE, Zaranek AW, Thakuria J, Wu X, et al: A highly annotated whole-genome sequence of a Korean individual. Nature 2009, 460:1011-1015.

13. Mardis ER, Ding L, Dooling DJ, Larson DE, McLellan MD, Chen $K$, Koboldt DC, Fulton RS, Delehaunty KD, McGrath SD, Fulton LA, Locke DP, Magrini VJ, Abbott RM, Vickery TL, Reed JS, Robinson JS, Wylie T, Smith SM, Carmichael L, Eldred JM, Harris CC, Walker J, Peck JB, Du F, Dukes AF, Sanderson GE, Brummett AM, Clark E, McMichael JF, et al: Recurring mutations found by sequencing an acute myeloid leukemia genome. $N$ Engl J Med 2009, 361:1058-1066.

14. Wang J, Wang W, Li R, Li Y, Tian G, Goodman L, Fan W, Zhang J, Li J, Guo Y, Feng B, Li H, Lu Y, Fang X, Liang H, Du Z, Li D, Zhao Y, Hu Y, Yang Z, Zheng H, Hellmann I, Inouye M, Pool J, Yi X, Zhao J, Duan J, Zhou Y, Qin J, Ma L, et al: The diploid genome sequence of an Asian individual. Nature 2008, 456:60-65.

15. Durbin RM, Abecasis GR, Altshuler DL, Auton A, Brooks LD, Gibbs RA, Hurles ME, McVean GA: A map of human genome variation from population-scale sequencing. Nature 2010, 467:1061-1073.

16. Goya R, Sun MG, Morin RD, Leung G, Ha G, Wiegand KC, Senz J, Crisan A, Marra MA, Hirst M, Huntsman D, Murphy KP, Aparicio S, Shah SP: SNVMix: predicting single nucleotide variants from next-generation sequencing of tumors. Bioinformatics 2010, 26:730-736.
17. Li R, Li Y, Fang X, Yang H, Wang J, Kristiansen K: SNP detection for massively parallel whole-genome resequencing. Genome Res 2009, 19:1124-1132.

18. Langmead B, Trapnell C, Pop M, Salzberg SL: Ultrafast and memoryefficient alignment of short DNA sequences to the human genome. Genome Biol 2009, 10:R25.

19. Li H, Durbin R: Fast and accurate short read alignment with BurrowsWheeler transform. Bioinformatics 2009, 25:1754-1760.

20. [http://www.novocraft.com].

21. Sherry S, Ward M, Kholodov M, Baker J, Phan L, Smigielski E, Sirotkin K: dbSNP: the NCBI database of genetic variation. Nucleic Acids Res 2001, 29:308-311.

22. Hubbard TJP, Aken BL, Ayling S, Ballester B, Beal K, Bragin E, Brent S, Chen Y, Clapham P, Clarke L, Coates G, Fairley S, Fitzgerald S, FernandezBanet J, Gordon L, Graf S, Haider S, Hammond M, Holland R, Howe K, Jenkinson A, Johnson N, Kahari A, Keefe D, Keenan S, Kinsella R, Kokocinski F, Kulesha E, Lawson D, Longden I, et al: Ensembl 2009. Nucl Acids Res 2009, 37:D690-697.

23. Montgomery SB, Sammeth M, Gutierrez-Arcelus M, Lach RP, Ingle C, Nisbett J, Guigo R, Dermitzakis ET: Transcriptome genetics using second generation sequencing in a Caucasian population. Nature 2010, 464:773-777.

24. Pickrell JK, Marioni JC, Pai AA, Degner JF, Engelhardt BE, Nkadori E, Veyrieras JB, Stephens M, Gilad Y, Pritchard JK: Understanding mechanisms underlying human gene expression variation with RNA sequencing. Nature 2010, 464:768-772.

25. Stranger BE, Forrest MS, Clark AG, Minichiello MJ, Deutsch S, Lyle R, Hunt S, Kahl B, Antonarakis SE, Tavare S, Deloukas P, Dermitzakis ET: Genome-wide associations of gene expression variation in humans. PLoS Genet 2005, 1:e78.

26. Dixon AL, Liang L, Moffatt MF, Chen W, Heath S, Wong KC, Taylor J, Burnett E, Gut I, Farrall M, Lathrop GM, Abecasis GR, Cookson WO: A genome-wide association study of global gene expression. Nat Genet 2007, 39:1202-1207.

27. Moffatt MF, Kabesch M, Liang L, Dixon AL, Strachan D, Heath S, Depner M, von Berg A, Bufe A, Rietschel E, Heinzmann A, Simma B, Frischer T, WillisOwen SA, Wong KC, Illig T, Vogelberg C, Weiland SK, von Mutius E, Abecasis GR, Farrall M, Gut IG, Lathrop GM, Cookson WO: Genetic variants regulating ORMDL3 expression contribute to the risk of childhood asthma. Nature 2007, 448:470-473.

28. Cookson W, Liang L, Abecasis G, Moffatt M, Lathrop M: Mapping complex disease traits with global gene expression. Nat Rev Genet 2009, 10:184-194.

29. Smyth GK: Linear models and empirical bayes methods for assessing differential expression in microarray experiments. Stat Appl Genet Mol Biol 2004, 3, Article3.

30. Venkatraman $E S$, Olshen $A B$ : A faster circular binary segmentation algorithm for the analysis of array CGH data. Bioinformatics 2007, 23:657-663.

31. Kanehisa M, Goto S, Furumichi M, Tanabe M, Hirakawa M: KEGG for representation and analysis of molecular networks involving diseases and drugs. Nucl Acids Res 2010, 38:D355-360.

32. Johnson DB: Efficient Algorithms for Shortest Paths in Sparse Networks. J ACM 1977, 24:1-13.

33. Fujita PA, Rhead B, Zweig AS, Hinrichs AS, Karolchik D, Cline MS, Goldman M, Barber GP, Clawson H, Coelho A, Diekhans M, Dreszer TR, Giardine BM, Harte RA, Hillman-Jackson J, Hsu F, Kirkup V, Kuhn RM, Learned K, Li CH, Meyer LR, Pohl A, Raney BJ, Rosenbloom KR, Smith KE, Haussler D, Kent WJ: The UCSC Genome Browser database: update 2011. Nucleic Acids Res 2010.

34. Koboldt DC, Chen K, Wylie T, Larson DE, McLellan MD, Mardis ER, Weinstock GM, Wilson RK, Ding L: VarScan: variant detection in massively parallel sequencing of individual and pooled samples. Bioinformatics 2009, 25:2283-2285.

35. Ramensky V, Bork P, Sunyaev S: Human non-synonymous SNPs: server and survey. Nucleic Acids Res 2002, 30:3894-3900.

36. Frazer KA, Ballinger DG, Cox DR, Hinds DA, Stuve LL, Gibbs RA, Belmont JW Boudreau A, Hardenbol P, Leal SM, Pasternak S, Wheeler DA, Willis TD, Yu F, Yang H, Zeng C, Gao Y, Hu H, Hu W, Li C, Lin W, Liu S, Pan H, Tang X, Wang J, Wang W, Yu J, Zhang B, Zhang Q, Zhao H, et al: A second 
generation human haplotype map of over 3.1 million SNPs. Nature 2007, 449:851-861.

37. Trapnell C, Pachter L, Salzberg SL: TopHat: discovering splice junctions with RNA-Seq. Bioinformatics 2009, 25:1105-1111.

38. Heap GA, Yang JH, Downes K, Healy BC, Hunt KA, Bockett N, Franke L, Dubois PC, Mein CA, Dobson RJ, Albert TJ, Rodesch MJ, Clayton DG, Todd JA, van Heel DA, Plagnol V: Genome-wide analysis of allelic expression imbalance in human primary cells by high-throughput transcriptome resequencing. Hum Mol Genet 2010, 19:122-134.

39. Yan XJ, Xu J, Gu ZH, Pan CM, Lu G, Shen Y, Shi JY, Zhu YM, Tang L, Zhang XW, Liang WX, Mi JQ, Song HD, Li KQ, Chen Z, Chen SJ: Exome sequencing identifies somatic mutations of DNA methyltransferase gene DNMT3A in acute monocytic leukemia. Nat Genet 2011, 43:309-315.

40. Robinson JT, Thorvaldsdottir H, Winckler W, Guttman M, Lander ES, Getz G, Mesirov JP: Integrative genomics viewer. Nat Biotechnol 2011, 29:24-26.

41. Nimer SD, MacGrogan D, Jhanwar S, Alvarez S: Chromosome 19 abnormalities are commonly seen in AML, M7. Blood 2002, 100:3838-3839, 3838 author reply.

42. Zhao Q, Kirkness EF, Caballero OL, Galante PA, Parmigiani RB, Edsall L, Kuan S, Ye Z, Levy S, Vasconcelos AT, Ren B, de Souza SJ, Camargo AA, Simpson AJ, Strausberg RL: Systematic detection of putative tumor suppressor genes through the combined use of exome and transcriptome sequencing. Genome Biol 2010, 11:R114.

doi:10.1186/1471-2105-12-267

Cite this article as: Deng: SeqGene: a comprehensive software solution for mining exome- and transcriptome- sequencing data. $B M C$ Bioinformatics 2011 12:267.

\section{Submit your next manuscript to BioMed Central} and take full advantage of:

- Convenient online submission

- Thorough peer review

- No space constraints or color figure charges

- Immediate publication on acceptance

- Inclusion in PubMed, CAS, Scopus and Google Scholar

- Research which is freely available for redistribution

Submit your manuscript at www.biomedcentral.com/submit
C Biomed Central 